PlastOx 2007 (2009) 265-275

(C) EDP Sciences, 2009

DOI: $10.1051 /$ ptox/2009019

\title{
Mécanismes et mécanique des interactions plasticité - environnements. Cas de l'industrie pétrolière
}

\author{
F. Ropital
}

IFP, Direction Chimie et Physico Chimie Appliquées, BP. 3, 69360 Solaize, France

e-mail : francois.ropital@ifp.fr

\begin{abstract}
Résumé. Les équipements de l'industrie pétrolières sont soumis à des environnements chimiques et à des conditions opératoires très variés. La maîtrise de leur intégrité est un enjeu majeur pour cette industrie. Les fluides transportés ou transformés peuvent contenir des agents corrosifs engendrant, par exemple, des fissurations sous contrainte et des cloquages des aciers au carbone. A hautes températures, les fortes activités en hydrogène ou en carbone sont à l'origine de dégradations par décarburation, metal dusting ou carburation des aciers faiblement alliés et des aciers inoxydables réfractaires. De plus les maintiens des ces aciers à hautes températures pendant des temps de fonctionnement très longs entraînent des évolutions microstructurales pouvant diminuer leur résistance aux différentes forme de corrosion. La compréhension et la modélisation des mécanismes de corrosion sont des axes importants pour la prédiction du comportement et la fiabilité des équipements de la chaîne pétrolière.
\end{abstract}

\section{INTRODUCTION}

La maîtrise de la corrosion des installations métalliques de l'industrie pétrolière est un enjeu majeur pour assurer son développement dans des conditions optimales de sûreté et de fiabilité. Afin de pouvoir répondre aux besoins sociétaux en carburants, l'industrie pétrolière doit maîtriser tous les processus de production, transport, raffinage, stockage des hydrocarbures. Ces processus ont lieu dans des environnements qui sont parfois spécifiques à cette industrie. La tableau 1 ci dessous donne une liste non exhaustive de certains milieux ainsi que des domaines pétroliers où ils sont présents.

Des connaissances fines des interactions entre d'une part les caractéristiques microstructurales et mécaniques des alliages métalliques et d'autre part les effets des environnements chimiques et sollicitations mécaniques sont essentielles pour une fiabilité maîtrisée des équipements tout en respectant les objectifs économiques. Les évolutions microstructurales apparaissant lors du vieillissement en service des matériaux peuvent fortement modifier leur comportement vis à vis de l'environnement et influer sur les paramètres de prédiction de la durée de vie des équipements.

Les paragraphes suivants vont présenter succinctement quelques exemples spécifiques à l'industrie pétrolières.

\section{ENVIRONNEMENTS LIQUIDES CORROSIFS}

\subsection{Fragilisation par l'hydrogène en milieu aqueux contenant de l' $\mathrm{H}_{2} \mathrm{~S}$}

Les phases aqueuses contenant du sulfure d'hydrogène sont à l'origine de deux phénomènes de dégradation des aciers au carbone et faiblement alliés et dans une plus faible mesure des aciers inoxydables: une corrosion généralisée de la surface et une fragilisation par l'hydrogène (FPH). Alors que pour le premier type de dégradation les vitesses de corrosion sont bien connues et faible du fait de la très bonne protectivité des sulfures de fer formés en phase aqueuse, le second type de dégradation est plus pernicieux. En effet le sulfure d'hydrogène est un promoteur puissant d'hydrogénation des aciers. 


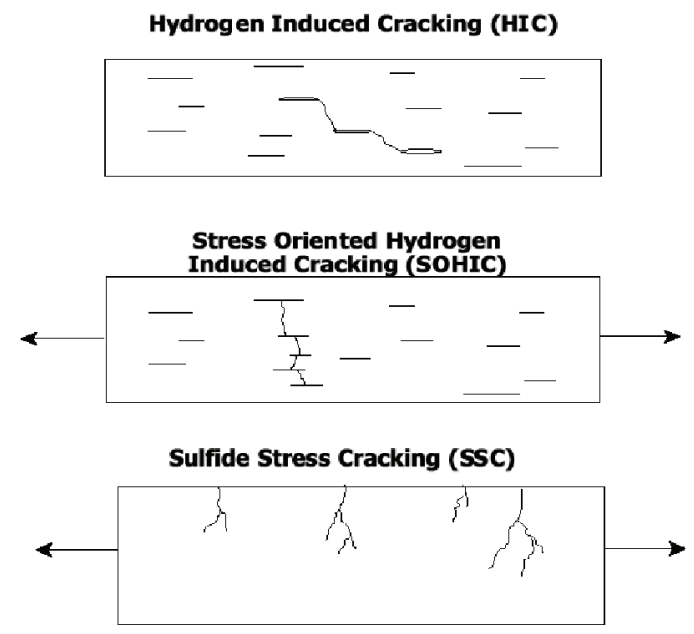

Figure 1. Schémas des dégradations HIC, SOHIC et SSC.

La présence d'hydrogène dissous dans un réseau métallique peut entraîner d'importantes détériorations du métal. Elles se caractérisent principalement par une chute des propriétés mécaniques entraînant la ruine de la structure, comme par exemple l'éclatement d'un pipeline.

$\mathrm{Du}$ point de vue phénoménologique, il est possible de distinguer deux types d'hydrogène : l'hydrogène interne et l'hydrogène externe.

Le terme hydrogène externe recouvre tous les phénomènes intervenant exclusivement sous chargement. Il est aussi dénommé SSC (Sulfide Stress Cracking) en milieu $\mathrm{H}_{2} \mathrm{~S}$ (figure 1).

Le terme hydrogène interne quant à lui recouvre tous les phénomènes pouvant également se dérouler après chargement; interviennent alors la décohésion interne par précipitation du gaz hydrogène en sursaturation sur certaines interfaces sensibles de la microstructure (notamment les bandes de perlite et les inclusions allongées de sulfure de manganèse), la fissuration en gradin ou le cloquage. Ces processus sont plus connus sous leur appellation anglaise respective de 'HIC' (Hydrogen Induced Cracking), 'SWC' (Step Wise Cracking) et 'blistering' (figure 1).

Des études récentes [1-3] ont bien mis en évidence ces deux types de fragilisation (par hydrogène interne et externe) et remis en cause le mode classique d'entrée de l'hydrogène dans l'acier par inhibition de la recombinaison des 2 atomes d'hydrogène pour donner le dihydrogène.

Tableau 1. Environnements corrosifs de l'industrie pétrolière (Légende: Pro. $=$ Production pétrolière, $\operatorname{Tr} .=$ Traitement de gaz, Raf. = Raffinage, Pétrochimie, CSC = Corrosion Sous Contrainte).

\begin{tabular}{|l|l|c|}
\hline Environnement corrosif & Mode de corrosion et matériaux concernés & Domaine Pétrolier \\
\hline eau liquide $+\mathrm{CO}_{2}$ & corrosion généralisée des aciers & Pro., Tr., Raf. \\
\hline eau liquide $+\mathrm{H}_{2} \mathrm{~S}$ & fragilisation par l'hydrogène des aciers & Pro., Tr., Raf. \\
\hline milieux alcalins & CSC des aciers au carbone & Pro., Tr., Raf. \\
\hline alcools (méthanol, éthanol) & CSC des aciers au carbone & Pro., Tr., Raf. \\
\hline solvants (amine) de gaz acides & CSC et généralisée des aciers & Tr., Raf. \\
\hline acides polythioniques & CSC aciers inoxydables sensibilisés & Raf. \\
\hline hydrogène en température & décarburation des aciers & Raf. \\
\hline oxygène, vapeur d'eau en température & oxydation des aciers et alliages & Raf. \\
\hline composés soufrés en température & sulfuration des aciers & Raf. \\
\hline composés carbonés en température & carburation, cokage, metal dusting & Raf. \\
\hline
\end{tabular}


Il est donc maintenant acquis de proposer que l'entrée d'hydrogène en présence d' $\mathrm{H}_{2} \mathrm{~S}$ correspond à l'entrée directe d'un proton sans étape de décharge. Ce processus très énergétique serait à la base du phénomène SSC. En effet, l'entrée du proton induit une contrainte de traction à la surface qui expliquerait l'observation de fissures dont l'amorçage serait à $90^{\circ}$ par rapport à la surface du matériau. Ainsi, l'arrêt du chargement (par émersion du matériau, par augmentation du pH de la solution ou encore par une diminution de la pression partielle d' $\mathrm{H}_{2} \mathrm{~S}$ ) stoppe immédiatement le SSC.

Pour le HIC, seul le $\mathrm{pH}$ [4] semble être le paramètre déterminant pour atteindre une pression interne en hydrogène donnée. Ceci peut se comprendre du fait de la relation entre le $\mathrm{pH}$ de la solution et l'activité du proton en solution en équilibre avec l'hydrogène dans le métal. Cependant la présence d'adsorbats sulfurés occasionne un flux plus grand et donc une mise à l'équilibre plus rapide qui peut même contribuer à fragiliser des métaux en permettant localement des sursaturations plus importantes.

La résistance des aciers à la FPH dépend de leur composition chimique, résistance mécanique et microstructure ainsi que de la sévérité du milieu aqueux hydrogénant.

Composition chimique : Le soufre, le phosphore et le manganèse sont reconnus comme les éléments les plus nocifs car ils ont une forte tendance à ségréger aux joints de grains où ils constituent des pièges pour l'hydrogène $[5,6]$. Une concentration plus élevée en $\mathrm{S}$ est favorable à la formation d'inclusions de $\mathrm{MnS}$ agissant comme des pièges à hydrogène. En revanche, les éléments carburogènes tels que le chrome, le molybdène et le vanadium augmentent la résistance à la FPH [7]. La précipitation fine de carbures dispersés de façon homogène dans la matrice permettrait de répartir de façon homogène la teneur volumique de l'hydrogène.

Résistance mécanique : Les aciers à haute résistance mécanique (résistance à la traction supérieure à $1000 \mathrm{MPa}$ ) sont reconnus comme étant les plus sensibles à la FPH [8]. En présence d'une grande quantité d'hydrogène, le mode de rupture de ces aciers est modifié et il devient de type fragile.

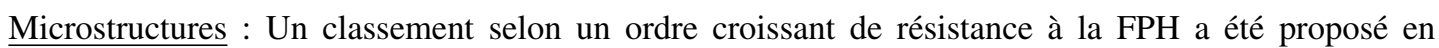
fonction de la microstructure des aciers [8]. Une structure fine présente la meilleure résistance à la FPH : Martensite brute $<$ Bainite brute $<$ Ferrite/Perlite $<$ Bainite revenue $<$ Martensite revenue

Zones de ségrégation (bandes de perlite, de bainite ou de martensite brute) : ce sont des sites privilégiés le long desquels se propagent des fissures en présence d'hydrogène [9]. En effet, la résistance mécanique plus élevée de ces zones fragilise le matériau. Les principaux éléments à considérer comme susceptibles de se ségréger, sont le phosphore, le soufre, le carbone, le manganèse et le nickel.

Inclusions non-métalliques : Ce sont des lieux privilégiés de nucléation des fissures en milieu $\mathrm{H}_{2} \mathrm{~S}$ [10]. La valeur de l'énergie d'interaction hydrogène/inclusions non-métalliques est un critère important pour évaluer l'effet de piégeage de l'inclusion.

Joints de grains : La résistance à la FPH des aciers diminue significativement si les joints de grain sont affaiblis par la ségrégation d'éléments néfastes tels que le phosphore et le soufre mais aussi l'étain, l'arsenic, l'antimoine.

Sévérité du milieu : La norme ISO 15156 [11] définit la sévérité des milieux $\mathrm{H}_{2} \mathrm{~S}$ (pression partielle $\mathrm{d}^{\prime} \mathrm{H}_{2} \mathrm{~S}$ et $\mathrm{pH}$ ) vis à vis de la fissuration de type SSC pour les aciers au carbone et aciers faiblement alliés en utilisant la figure 2. Ce diagramme a été obtenu suite à de nombreux travaux expérimentaux mais aussi grâce aux retours d'expériences sur champs pétroliers.

A partir de ces paramètres, nous constatons que la compréhension des mécanismes de FPH fait appel à une connaissance fine des interactions entre l'hydrogène, la microstructure et les propriétés mécaniques des aciers. 


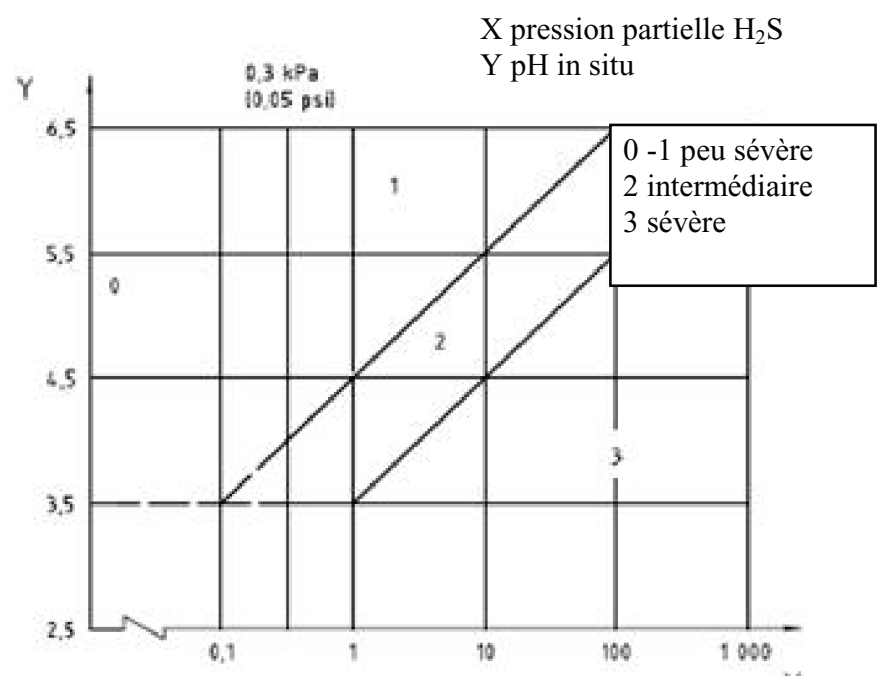

Figure 2. Sévérité des milieux $\mathrm{H}_{2} \mathrm{~S}$ vis à vis de la fissuration SSC selon la norme ISO 15156 [11].

\subsection{Corrosion sous contrainte des aciers en milieux alcalins}

Les milieux alcalins sont bien connus pour pouvoir engendrer des fissurations des aciers dans des zones à faible plasticité et ou fortement sollicitées mécaniquement. Les principaux environnements alcalins rencontrés dans le domaine pétroliers sont des solutions de soude ou de carbonates.

Corrosion sous contrainte en milieux caustiques : Des bases comme $\mathrm{NaOH}$ et parfois $\mathrm{KOH}$ sont utilisées dans de nombreux procédés de raffinage et de traitement de gaz. Ces bases peuvent, même à faible concentration (50-100 ppm), engendrer des dégradations par corrosion sous contrainte des aciers au carbone, des aciers faiblement alliés et de certains aciers inoxydables austénitiques. Les principaux équipements concernés sont les zones dans lesquelles la soude (ou la potasse) est injectée pour neutraliser des effluents acides ainsi que les chaudières et générateurs de vapeur.

Cette corrosion sous contrainte a un mode de propagation principalement intergranulaire; toutefois quelques cas de cheminement transgranulaire sont reportés dans la littérature [12].

Les principaux paramètres qui gouvernent cette corrosion sont la concentration en agent caustique, la température et les contraintes appliquées ou résiduelles que subissent l'acier.

Corrosion sous contrainte en milieux carbonatés : Des phénomènes de corrosion sous contrainte des aciers au carbone et faiblement alliés sont de plus en plus souvent rencontrés dans des unités FCC (Fluid Catalytic Cracking) pour lesquelles de l'eau libre contenant des ions carbonates - bicarbonates, de l' $\mathrm{H}_{2} \mathrm{~S}$ et de l'ammoniac peut être présente [13].

Des cas de corrosion sous contrainte en milieux carbonatés ont été rapportés pour des unités de traitement de gaz contenant plus de $2 \%$ de $\mathrm{CO}_{2}$ et pour des températures de plus de $90{ }^{\circ} \mathrm{C}$ [13].

Cette corrosion sous contrainte présente un cheminent intergranulaire de fines fissures remplies d'oxydes. Elle apparaît dans des zones sous contrainte; pour les soudures les fissures se propagent parallèlement à la soudure dans les zones de faible plasticité (fortes contraintes résiduelles mais également dans les zones thermiquement affectées) [10, 13-15].

Les principaux paramètres gouvernant cette corrosion sont: le $\mathrm{pH}$ de l'eau libre résiduaire (du à l'ammoniac), la composition de l'eau (concentration en carbonates, $\mathrm{H}_{2} \mathrm{~S}$, cyanures), les contraintes résiduelles, le potentiel électrochimique d'abandon de l'acier. 


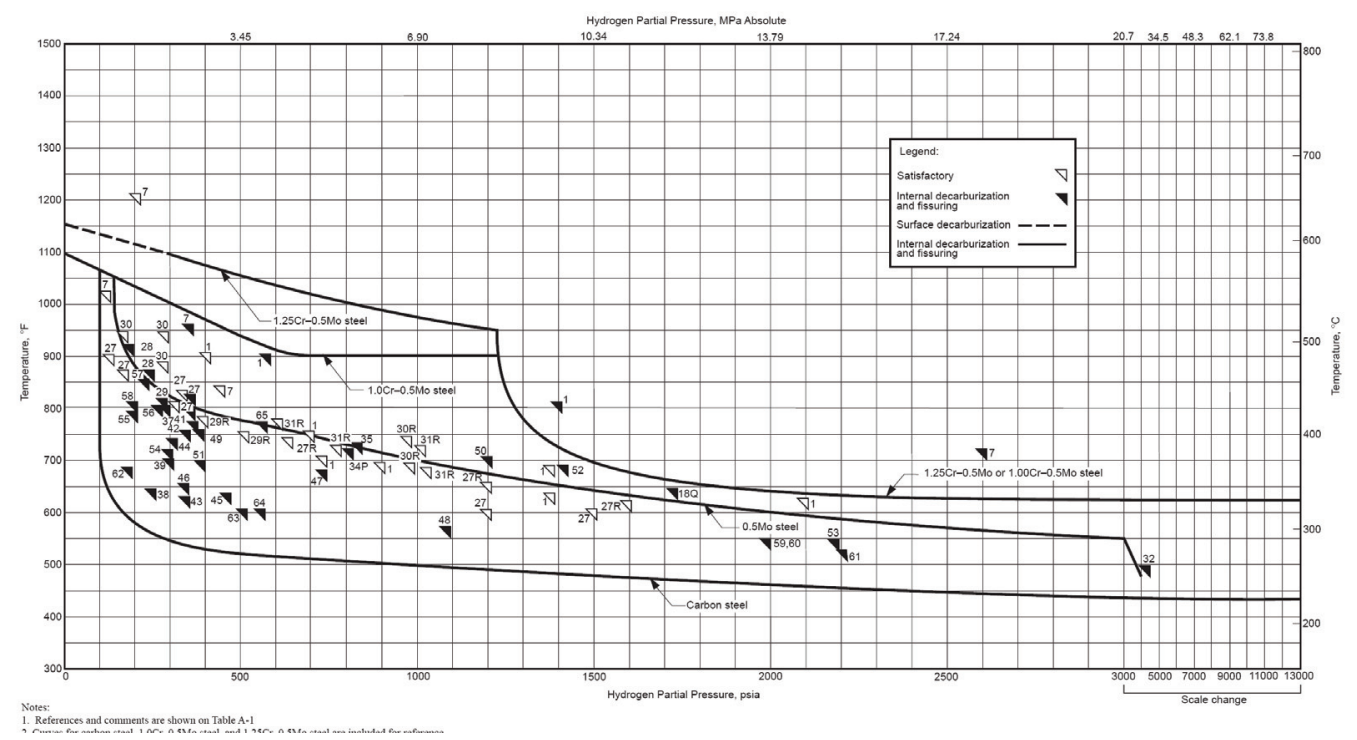

Figure 3. Courbe de l'acier 0, 5\% Mo de 1997 avec les cas de dégradation observés (extrait API RP 941-2004).

Ces phénomènes de CSC peuvent se rencontrer pour les conditions suivantes de basicité de l'eau libre [13-15] :

- $\mathrm{pH} \geq 7,6$ à partir d'une concentration en $\mathrm{H}_{2} \mathrm{~S}$ d'au moins $50 \mathrm{ppm}$

- $8 \leq \mathrm{pH} \leq 9$ à partir d'une concentration en carbonate d'au moins $400 \mathrm{ppm}$

- $\mathrm{pH} \geq 9$ à partir d'une concentration en carbonate d'au moins 100 ppm.

Le potentiel pour lequel cette corrosion intervient est en général compris entre -500 et $-650 \mathrm{mV} / \mathrm{ECS}$. Pour les solutions aqueuses contenant de $\mathrm{l}^{\prime} \mathrm{H}_{2} \mathrm{~S}$, la présence d'ions $\mathrm{CN}^{-}$en limitant la formation de la couche de sulfure de fer pourrait influer sur le potentiel d'abandon en l'amenant dans le domaine de sensibilité à la corrosion sous contrainte.

Ces phénomènes de CSC ont fait l'objet de nombreux travaux de recherche et pour s'en prémunir des traitements thermiques de détensionnement sont préconisés et, lorsque que le risque a été identifié, appliqués. Toutefois des retours d'expériences industrielles de rupture d'équipements de FCC détensionnés [16], indiqueraient que des connaissances plus fines des relations plasticité - fragilisation alcaline semblent nécessaires.

\subsection{Corrosion sous contrainte des aciers au carbone par le méthanol ou l'éthanol}

Les alcools à chaîne carbonée courte comme le méthanol et l'éthanol peuvent être à l'origine de corrosion sous contrainte des aciers au carbone. Le méthanol peut également engendrer des ruptures par corrosion sous contrainte du titane et de ses alliages (et notamment l'alliage Ti-6Al-4V). Dans la production pétrolière, le méthanol est utilisé comme agent thermodynamique de prévention de la formation des hydrates de gaz. Les équipements de stockage et de transport de cet agent chimique en acier au carbone et en alliage de titane sont donc concernés. Des mélanges réfrigérés méthanol - eau sont utilisés comme solvant pour l'extraction des gaz acides $\left(\mathrm{CO}_{2}, \mathrm{H}_{2} \mathrm{~S}\right)$ dans certains procédés de traitement de gaz. Les réservoirs et lignes de transport sont en acier au carbone et sont potentiellement concernés. Dans l'industrie chimique et du raffinage, le méthanol est utilisé pour la fabrication du MethylTertButylEther (MTBE) par réaction avec de l'isobutylène. Les réservoirs en acier au carbone de ces unités peuvent être affectés. 
L'éthanol est quant à lui employé comme carburant de substitution : les réservoirs, cuves et pipes en acier au carbone sont plus particulièrement concernés par une possible corrosion sous contrainte. Du fait du fort développement des bio carburants cette problématique est tout à fait d'actualité.

A notre connaissance, peu de travaux ont été réalisés sur les mécanismes d'endommagement par ces alcools en relation avec les propriétés mécaniques des aciers. Toutefois les éléments présentés ci dessous permettent de cerner certains paramètres.

Corrosion sous contrainte de l'acier au carbone par le méthanol : La présence d'eau et de certains composés chimiques (acide formique, chlorures) dans le méthanol est la cause principale des phénomènes de fissuration sous contrainte des aciers au carbone [17-22]. La propagation de la fissuration est le plus souvent intergranulaire.

Les principaux paramètres responsables de la corrosion sous contrainte des aciers au carbone sont $[17,20]$ :

- la teneur en eau : les conditions de corrosion sont rapportées dans la littérature pour des teneurs en eau inférieures à $1 \%$, la sensibilité étant la plus forte dans le domaine $0,05-0,5 \%$,

- l'acide formique (produit de dégradation du méthanol) lorsqu'il est présent jusqu'à 300 ppm, accélère la CSC,

- lorsqu'ils sont présents les ions chlorures participent à la déstabilisation de la couche initiale d'oxyde protectrice,

- la présence d'oxygène pourrait aggraver les risques de corrosion sous contrainte, en amenant l'acier dans un domaine de le domaine de potentiel de sensibilité SCC. De plus une influence de l'oxygène sur la dégradation des alcools en éléments corrosifs (acide formique) n'est pas à exclure.

Pour les alliages de titane, la corrosion sous contrainte a un cheminement intergranulaire qui est favorisée par la présence d'ions halogénures. La présence d'une quantité suffisante d'eau (de 2 à 20\%) permettrait de s'affranchir de la CSC des alliages de titane [22-24].

\section{ENVIRONNEMENTS GAZEUX À HAUTES TEMPÉRATURES}

\subsection{Attaque par l'hydrogène à hautes températures}

Les matériaux en contact avec hydrogène à hautes températures doivent être résistants face à l'attaque par l'hydrogène. Tous les procédés de raffinage sous hydrogène sont concernés, en particulier les unités d'hydrotraitement et d'hydrocraquage.

Si la spécification matériau n'est pas adaptée, l'hydrogène peut entraîner la décarburation du métal. En effet, en opération, l'hydrogène se recombine avec les carbures (cémentite $\mathrm{Fe}_{3} \mathrm{C}$ ) de l'acier pour former du méthane ce qui, d'une part fait chuter les caractéristiques mécaniques du métal et d'autre part peut être la cause de fissurations (le méthane, ne pouvant pas diffuser, peut provoquer des fissurations intergranulaires).

Les matériaux pouvant être affectés par ce phénomène sont les aciers carbones, et les aciers faiblement alliés.

L'attaque par hydrogène à chaud se produit sous 2 formes :

- la décarburation de surface

- la décarburation interne

Les fortes températures et faibles pressions partielles d'hydrogène favorisent le premier mode, tandis que le second mode se développe surtout à des températures plus modérées sous forte pression partielle d'hydrogène.

Décarburation de surface : Le mécanisme généralement admis repose sur la migration du carbone vers la surface où il se combine avec l'hydrogène pour former un composé gazeux. Contrairement à la 


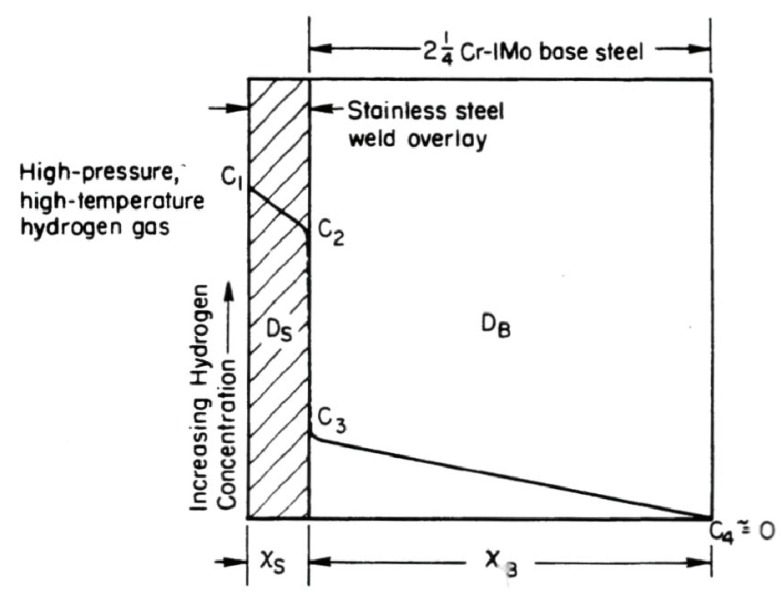

Figure 4. Schéma montrant la distribution de l'hydrogéne dans la paroi d'un équipment en acier ferritque avec un overlay en acier ausénitique.

décarburation interne, la décarburation de surface ne se traduit pas par l'apparition de microfissures, mais par une réduction de la résistance mécanique et un accroissement de la ductilité.

Décarburation interne : La décarburation interne est liée à la diffusion de l'hydrogène dans l'acier et sa réaction avec le carbone pour donner du méthane. Le méthane formé ne peut diffuser hors du métal et tend à s'accumuler aux joints de grain. Ceci conduit à des contraintes importantes dans le métal provoquant la formation de micro vides, puis de micro fissures, qui se rejoignent pour former des fissures ou des blisters selon le cas.

Pour éviter la formation de méthane, les choix métallurgiques conduisent à la substitution des carbures de fer par des carbures plus stables vis à vis de l'hydrogène tels que les carbures de chrome, de molybdène, de titane, de vanadium ou de niobium (éléments d'addition des aciers alliés). Dans les domaines de sensibilité, les aciers faiblement alliés voire les aciers inoxydables sont donc choisis.

Les courbes dites de Nelson de la norme API 941 indiquent les domaines d'utilisation des aciers carbones et faiblement alliés de type Cr-Mo. Les limites d'utilisation sont des fonctions de la pression partielle d' $\mathrm{H}_{2}$ et de la température. Ces courbes sont basées sur des retours d'expériences et sont régulièrement mises à jour.

Disbonding des revêtements : Certains équipements opérant sous pression d'hydrogène en température bénéficient d'un revêtement interne protecteur en acier inoxydable austénitique. Ces revêtements sont soit réalisés par colaminage, plaquage par explosion, soudage ou même doublage. Ils sont utilisés sur les unités d'hydrodésulfuration ou le métal de base est choisi pour sa résistance à l'attaque par hydrogène à chaud et ses caractéristiques mécaniques à température élevée. Le revêtement assure une bonne tenue à la sulfuration à chaud.

Le disbonding est un phénomène de décollement de ce revêtement austénitique. En effet il existe une forte différence de solubilité et de diffusion de l'hydrogène : le coefficient de diffusion de l'hydrogène dans les aciers austénitiques est très inférieur à celui des aciers ferritiques. Il en résulte une sursaturation de l'hydrogène à l'interface et une possible décohésion du revêtement de l'acier ferritique de base (figure 4). Afin d'évaluer la sensibilité des revêtements à ces phénomènes de décollement, des essais de laboratoire, pour lesquels les conditions sont déterminées par simulations numériques [25] peuvent être réalisés. 

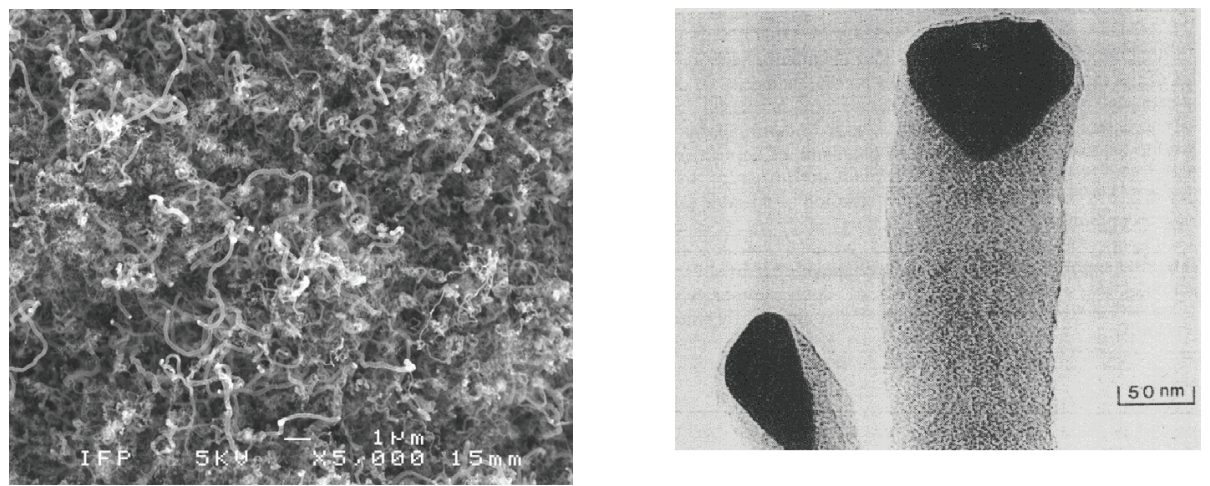

Figure 5. Images de filaments de coke catalytique.

\subsection{Attaques par les atmosphères carbonées à hautes températures}

Dans les procédés de raffinage du pétrole, la transformation des charges en coupes de compositions ciblées d'hydrocarbures est réalisée sous des atmosphères de fortes activités en carbone, engendrant différentes formes d'attaque des équipements métalliques.

Cokage et Metal dusting : En fonction du domaine de température, plusieurs mécanismes sont la cause des dépôts de carbone appelés coke à la surface de nombreux métaux et alliages. Ce coke provient de la décomposition des hydrocarbures.

mécanisme hétérogène catalytique entre 400 et $900^{\circ} \mathrm{C}$ : un réseau de filaments de carbone (nanotubes) est catalysé par le fer et (ou) le nickel présents à la surface des tubes et réacteurs. Des molécules d'hydrocarbure sont chimiesorbées sur des cristallites métalliques. La formation de ces cristallites de métal ou "dust" conduit à l'attaque localisée et parfois généralisée de l'équipement par "metal dusting". Malgré leur taille nanométrique, du fait de l'arrachage d'un grand nombre de particules et de la carburation en surface associée, ce phénomène peut provoquer des pertes d'épaisseur significatives et des percements des parois.

mécanismes hétérogènes non catalytiques entre 500 et $900^{\circ} \mathrm{C}$ : les réactions de formation du coke sont générées par des précurseurs de coke (comme les composés aromatiques et insaturés) sur des sites actifs de la surface.

Sur une surface de fer ou d'acier oxydée, les oxydes de fer présents à la surface de l'échantillon favorisent la formation du coke (figure 6). Au contact du mélange réactionnel, la couche d'oxyde se fragmente et se transforme en particules catalytiques. Le diagramme de stabilité de phases du système $\mathrm{Fe}-\mathrm{C}-\mathrm{O}$ montre que la magnétite et la wüstite en présence d'un mélange réducteur et carburant peuvent être directement transformées en carbure sans passer par un état réduit du fer. Les particules catalytiques sont constituées d'une solution solide de fer sursaturée en carbone recouverte d'une couche de cémentite [26].

mécanismes homogènes non catalytiques pour des températures supérieures à $700^{\circ} \mathrm{C}$ : ce sont des réactions radicalaires en phase gazeuse qui conduisent à la formation de composés polyaromatiques de poids moléculaire élevé. Ces mécanismes pyrolitiques apparaissent principalement dans les fours de vapocraquage.

Carburation : Le carbone du coke qui se dépose à la surface des métaux peut diffuser à l'intérieur de l'alliage où il se retrouve en solution dans la matrice (jusqu'à sa limite de solubilité) ou sous forme de carbures localisés principalement aux joints de grains. La présence d'une couche superficielle d'oxydes imperméable peut être bénéfique car elle limite la pénétration du carbone. Ces couches se forment difficilement lorsque $\mathrm{Po}_{2}$ est très faible. A l'opposé de l'action de l'hydrogène à chaud, cet apport de 


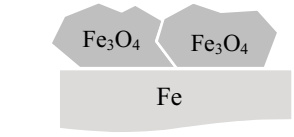

-1- Etat Initial

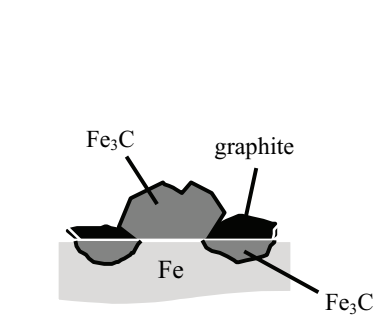

-4- Graphitisation de la surface

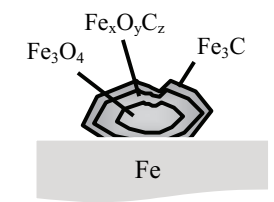

-2- Carburation et fragmentation de l'oxide $\left(\mathrm{VFe}_{3} \mathrm{O}_{4}>\mathrm{VFe}_{3} \mathrm{C}\right)$

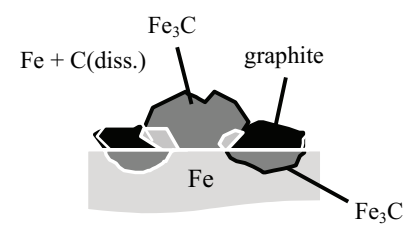

-5- Decomposition de la cémentite

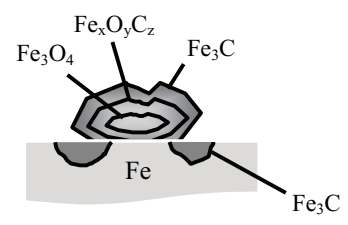

-3- Carburation du fer

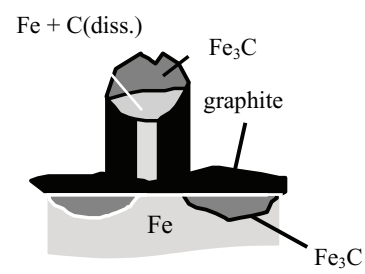

-6- Croissance du filament

Figure 6. Mécanisme de dépôt de coke sur une surface de fer oxydée [26].

carbone va fortement diminuer la plasticité de l'alliage voire totalement supprimer toute aptitude à la déformation plastique. Le comportement mécanique du matériau est alors fortement affecté devenant très sensible aux variations thermiques.

\section{VIEILLISSEMENT ET RÉSISTANCE À LA CORROSION}

Les équipements du raffinage et de la pétrochimie sont conçus pour de très longues durées de service ( $>10$ ans) en température. Pour certains matériaux métalliques, ces maintiens prolongés en température peuvent engendrer des phénomènes de vieillissement se traduisant par des modifications locales de leur microstructure (sensibilisation, précipitation de phases intermétalliques) et de leur caractéristiques mécaniques. Ces transformations peuvent parfois avoir une incidence sur la résistance chimique du matériau à l'environnement. Quelques exemples sont donnés ci après.

\subsection{Aciers inoxydables sensibilisés et corrosion par les acides polythioniques}

Les acides polythioniques $\left(\mathrm{H}_{2} \mathrm{~S}_{\mathrm{x}} \mathrm{O}_{6}\right)$ se forment pas oxydation des composés soufrés à température ambiante et en présence d'humidité et d'air (typiquement formés lors des phases d'arrêt des unités).

Ces acides peuvent être la cause de corrosion sous contrainte importante des aciers inoxydables si ces derniers ont été sensibilisés par précipitation de carbures de chrome au dessus de $400^{\circ} \mathrm{C}$. Si le risque existe, il est généralement recommandé de spécifier des aciers inoxydables stabilisés par des éléments d'addition carburigènes tels que le titane ou le niobium.

\subsection{Fragilités des aciers}

La fragilité de revenu réversible (FRR) est un phénomène de vieillissement qui apparaît pour les aciers faiblement alliés au Cr-Mo particulièrement le $21 / 4 \mathrm{Cr}-1$ Mo trempé et revenu, pour le domaine de température de $375-550{ }^{\circ} \mathrm{C}$ avec une cinétique maximale vers $470{ }^{\circ} \mathrm{C}$.

Le temps d'incubation est généralement long: il peut diminuer dans le cas de matériaux très sensibles (matériaux par exemple avec $\mathrm{P}$ élevé $>0.015 \%$ ). La fragilisation est provoquée par la ségrégation 
d'éléments résiduels au niveau des joints de grains austénitiques, provoquant une chute de la ductilité et une augmentation de la température de transition ductile / fragile.

Creep embrittlement : Les aciers faiblement alliés au Cr-Mo, particulièrement les $1 \mathrm{Cr}-1 / 2 \mathrm{Mo}$ et $11 / 4 \mathrm{Cr}$ -

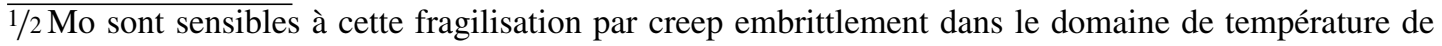
fluage. Des cavités et fissures secondaires se développent aux joints de grains de l'austénite. Une taille de grain élevée (du fait d'énergies de soudage trop importantes) et des concentrations de contrainte sont les principaux facteurs de fragilisation. Cet endommagement n'est détecté qu'après plusieurs années ( $\geq 8$ ans) engendrant, par baisse de la ductilité, des fissurations dans les zones affectées thermiquement et également dans les soudures.

\section{CONCLUSION}

Comme cela vient d'être présenté à l'aide de quelques exemples, les environnements chimiques de l'industrie pétrolière interagissent fortement avec les caractéristiques mécaniques globales et locales des alliages métalliques. Pour certains milieux, de nombreux travaux ont été réalisés pour mieux comprendre les mécanismes d'endommagement et mieux les prédire : fissurations en milieux alcalins, fragilisation par l'hydrogène, attaques à hautes températures par l'hydrogène ou le carbone. Toutefois les mutations de l'industrie pétrolière la conduisent à s'intéresser à d'autres milieux comme ceux de la biomasse et des biocarburants à fortes teneurs en éthanol, pour lesquels le champ d'investigation des interactions entre l'environnement et les propriétés mécaniques est encore à approfondir.

\section{Références}

[1] Crolet J.L., Maisonneuve G.G., Eurocorr 2000 (London UK, The institute of Materials, 2000).

[2] Crolet J.L., Bonis M., NACE Corrosion 2001, paper 067.

[3] Duval S., Antono-Lopez R., Scomparin M., Jérome M., Ropital F., NACE Corrosion 2004, paper 740.

[4] Baethmann H.J., Graef M., Hoh B., Kleinen J. et Poerpperling R., NACE Corrosion 84, paper 003.

[5] Ikeda A., Secon Int. Conf. Interaction of Steels with Hydrogen in Petroleum Industry Pressure Vessel and Pipeline Service, Wien, Autriche, (1994) p. 161.

[6] Bowker J., Piggot M.R., Weatherly G.C., VI. Int. Conf. On Fracture, New Delhi, India, Vol. 4, (1984) p. 2371.

[7] Ravi K., Ramaswamy V., Namboodhiri, T.K.G. Mat. Sci. Eng. A 169 (1993) 111.

[8] Biagiotti P.E. et al., NACE Corrosion 95, paper 64.

[9] Blondeau R., Le livre de l'acier, Lavoisier Ed., Paris, (1994) p. 407.

[10] Mirabal E., Bhattacharjee S., Pazos N., NACE Corrosion 1991, paper no. 312.

[11] Norme ISO 15156 "Petroleum and natural gas industries : materials for use in H2S containing environments in oil and gas production.

[12] NACE Recommended Practice 0403 (2003 Revision).

[13] Rivera M., Bolinger S., Wollenweber C., NACE Corrosion 2004, paper no. 04639.

[14] Kmetz J.M., Truax D.J., NACE Corrosion 1990, paper no. 206.

[15] Parkins R.N. et al., S. Bolinger, C. Wollenweber, NACE Corrosion1986, paper no. 205.

[16] Nace technical Committee "Review and Survey of Alkaline Carbonate Stress Corrosion Cracking in Refinery Sour Waters" Report TG347 draft version (2007).

[17] Kefferstein R., Jartoux X., La Revue de Métallurgie 3 (1998) 389.

[18] Bellucci F. et al., Corrosion Science, 28 n4, (1988) 371.

[19] Farina C.A. et al., Corrosion Science, 18, 465.

[20] API Technical Report 939-D, September 2003. 
[21] Kane R.D., Maldonado J.G., Klein L.J., NACE Corrosion 2004, paper no. 04543.

[22] Chapter on Corrosion of Titanium and Titanium Alloys, Metals Handbook, Vol. 13, Corrosion, ASM International (1987) pp. 687.

[23] Sergent E., Roques Y., Corrosion Science, 36 (1994) 653.

[24] Schutz R.W., Horrigan J.M., Bednarowicz T.A., NACE Corrosion 1998, paper no. 261.

[25] Coudreuse L., Cadilhac B., Dumontier C., Koers R., Riccardi G., Roche M., Ropital F., Eurocorr 1999 Conference (1999) paper 320.

[26] Bonnet F., Ropital F., Berthier Y., Marcus P., Materials and Corrosion, 54 (2003) 870. 
